\title{
Die Dynamik des Konsums und die moralische Integration moderner Gesellschaften - ein Konzeptualisierungsversuch ${ }^{1}$
}

\section{Der moderne Erlebniskonsum: eine sozial integrierende oder zerstörende Kraft?}

In meinem Text soll es um die »moralischen « Seiten des Konsums gehen, das heißt um die Frage, ob und welchen Beitrag der moderne, dynamische Erlebniskonsum für die soziale »Integration ", den viel zitierten "Zusammenhalt « der Gesellschaft, leistet. Diese Frage ist in der Konsum- und Kultursoziologie kontrovers diskutiert worden. Zunächst scheint es notwendig, die Fragestellung näher zu erläutern, denn dass der Konsum überhaupt "moralische " Funktionen erfüllt, ist unmittelbar keineswegs einsichtig. Vor allem in den Wirtschaftswissenschaften wird Konsum noch immer gern mit dem »materiellen " Verbrauch gleichgesetzt. Konsumgüter und -dienstleistungen werden als Mittel zur Befriedigung von »Bedürfnissen " verstanden, die letztlich in der physischen Natur des Menschen wurzeln und als solche eine exogene, wirtschaftsexterne Größe sind. Die Menschen müssen essen, trinken, sich kleiden, wohnen, reisen usw., und die Wirtschaft stellt die Mittel bereit, um diese Bedürfnisse reichlich und immer komfortabler zu befriedigen. Konsum hat in dieser Sicht nur eine ökonomische, keine moralische Bedeutung.

Der Hinweis auf die Materialität des Konsums ist nicht falsch. Der alleinige Fokus auf sie ist aus soziologischer Sicht jedoch verkürzt, denn der Konsum hat nicht nur eine materielle, sondern immer auch eine symbolische Seite. Konsumgüter »sind « nicht nur etwas, sondern »bedeuten " gleichzeitig etwas, sind Träger kultureller Verweisungen. ${ }^{2}$ Da Kulturen sozial differenziert sind, senden die Konsumenten durch die Art, wie sie sich kleiden, wohnen, den Urlaub verbringen usw., ebenso soziale Signale über ihre gesellschaftliche Position aus. Dieser »symbolische« Konsum bestimmt heute den überwiegenden Teil der Konsummärkte. In engem Zusammenhang damit steht der dynamische, durch Modeabhängigkeit und ständigen Veränderungsdruck geprägte Charakter dieser Märkte. Ginge es beim Konsum nur um die Befriedigung physischer Bedürfnisse, so wäre es mit dem Wachstum der Märkte bald zu Ende, denn physische Bedürfnisse sind ihrer Natur nach begrenzt. Im Gegensatz dazu scheinen der symbolische Konsum und die durch ihn hervorge-

1 Dieser Aufsatz ist während eines Aufenthalts des Autors als Fellow am Internationalen Forschungszentrum Kulturwissenschaften in Wien (IFK) im Sommer 2012 geschrieben worden. Ich danke Helmut Lethen, Lutz Musner, den MitarbeiterInnen des Instituts sowie meinen MitstipendiatInnen für die Gastfreundschaft und die anregenden Diskussionen am Institut. Eine erste Version des Aufsatzes habe ich auch am Institut für Soziologie der Universität Graz vorgetragen.

2 Vgl. Douglas, Isherwood 1996 [1979]; McCracken 1988.

Leviathan, 40. Jg., 4/2012, S. 5177 535 
brachten immer neuen Wünsche in der Tat »unersättlich « zu sein, und so kommt es zur Entstehung stets neuer Produkte, Dienstleistungen und Moden; die Märkte für Unterhaltungselektronik sind vielleicht das eindrucksvollste neuere Beispiel dafür.

In einem gewissen Sinne ist die Dynamik des Konsums, wie Baudrillard ${ }^{3}$ betont hat, in seinem symbolischen Charakter selbst bereits angelegt. Die Bedeutung von Zeichen ergibt sich erst aus ihrer Differenz zu anderen Zeichen, und so treten mit der Erfüllung jedes Konsumwunsches immer neue Möglichkeiten und Wünsche in den Blick, die nach Befriedigung verlangen. Durch die Art, wie sie essen, wohnen, sich kleiden usw., senden die Konsumenten Botschaften konträrer Art aus. Einerseits geben sie Auskunft über die sozialen Milieus und Schichten, denen sie sich zurechnen. Sie reproduzieren die für jene Milieus charakteristischen alltagsästhetischen Schemata und mit ihnen die Milieus selbst, deren Selbstbild seinerseits zu einem wichtigen Teil durch Konsumpraktiken bestimmt wird. ${ }^{4}$ Werbung, Design und Markenpolitik sind bemüht, Produkte und Dienstleistungen mit kulturellen Bedeutungen aufzuladen und so ihren Identifikationswert absatzfördernd zu erhöhen. ${ }^{5}$ Dabei können die Marketing-Strategen der Industrie freilich nicht nur auf etablierte, schicht- beziehungsweise milieuspezifisch differenzierte kulturelle Muster zurückgreifen, sondern auch neue Moden initiieren und so die Marktdynamik vorantreiben. ${ }^{6}$ Auf der anderen Seite möchten die Konsumenten sich selbst in einer bestimmten Weise präsentieren, ihre persönlichen Eigenschaften jenseits der bloßen Milieu- und Schichtzugehörigkeit in ein positives Licht rücken. Sie wollen sich nicht nur so darstellen, wie sie sind, sondern auch, wie sie sein möchten. Sie realisieren die herrschenden Moden nicht eins zu eins, sondern setzen bei ihrer Rezeption individuelle Akzente und versuchen, daraus einen individuellen Distinktionsgewinn zu erzielen. ${ }^{7}$ In diesem Sinn signalisiert der Konsum nicht nur kollektive, sondern auch individuelle Identitäten. Die Dynamik des Konsums ergibt sich aus diesem immerwährenden Wechsel zwischen den Inszenierungen: Vertrautes gegen Neues, individuelle gegen kollektive Identitäten.

Die Symbolik des Konsums als solche ist nichts spezifisch Modernes. Auch in ständischen Gesellschaften und sogar in Stammesgesellschaften ist der Konsum niemals bloß " physischer "Verbrauch, sondern immer in symbolische und soziale Ordnungen eingebettet und dient der Reproduktion dieser Ordnungen. Die Ordnungen wurden jedoch - trotz begrenzter Statuskämpfe, die es immer gab - im Kern als etwas Unveränderliches erfahren. Jeder Mensch hatte seinen Platz in der als naturoder gottgegeben betrachteten Sozialhierarchie, und dieser Platz wurde durch die Art der Kleidung, Nahrung, Möblierung usw. markiert. »Wer mehr konsumierte als ihm standesgemäß zustand, störte diese Ordnung und geriet in den Geruch der

\section{Baudrillard 1998.}

4 Hier ist auf die einschlägigen Analysen Bourdieus zu verweisen.

5 Hellmann 2003.

6 McCracken 1988, S. $71 \mathrm{f}$.

7 Simmel 1995 [1905]. 
Sünde «, 8 stellt Jakob Tanner fest. Das heißt, der Konsum erfüllte nicht nur die Funktion materieller Reproduktion, sondern brachte auch die soziale Identität und moralische Konformität der Individuen zum Ausdruck und trug so gesehen zur Integration der Gesellschaft bei; ständische Konsumnormen sind »soziale Tatsachen « im Durkheim'schen Sinn.

Der moderne Konsum setzte mit den » bürgerlichen « Konsumrevolutionen des 17. und 18. Jahrhunderts (vor allem in den Niederlanden und in Großbritannien) ein. ${ }^{9}$ Vor dem Hintergrund steigender Geldeinkommen und fortschreitender Warenförmigkeit der Konsumgüter ${ }^{10}$ strebten bürgerliche Aufsteiger danach, mit ihrem Konsumverhalten nicht nur Konformität, sondern auch Differenz zu markieren. Indem sie sich die Statussymbole höherer Schichten aneigneten, durchbrachen sie systematisch die ständischen Codes und setzten einen Prozess beständiger Veränderung dieser Codes selbst in Gang. Die Konsumsphäre wie auch die Kunst wurden damit zum Schauplatz konkurrierender Inszenierungen etablierter kultureller Normen und individueller Differenzansprüche gegen diese Normen. Es entwickelte sich das oben beschriebene Konkurrenzverhältnis gegenläufiger sozialer Signale - ein Konkurrenzverhältnis, das sich nur in der Zeit, das heißt dynamisch entfalten kann. Nicht die soziale Symbolik des Konsums als solche, sondern die Dynamisierung dieser Symbolik kann als Spezifikum des modernen Konsums betrachtet werden.

Welches sind die Rückwirkungen der modernen Dynamisierung des Konsums auf seine sozial integrativen Funktionen? Hier nun gehen die Meinungen weit auseinander. Viele, teils aus der Konsumsoziologie, teils aus der Religionssoziologie kommende Autoren argumentieren, dass der Konsum gerade dank seiner Dynamisierung einen entscheidenden Beitrag zur sozialen Kohärenz der modernen Gesellschaft leistet (a). Eine entschiedene Gegenposition dazu hat Daniel Bell vertreten (b).

(a) Die meisten Konsumsoziologen sind heute der Meinung, dass der Konsum gegenwärtig über seine ökonomische Bedeutung hinaus »soziale Identitäten « vermittelt. Mit dem auf George H. Mead zurückgehenden Konzept sozialer Identität sind kollektive Selbstdeutungen gemeint, die das Individuum zu einem mit diesen Deutungen konformen Verhalten verpflichten. Maßgeblich dabei sind nicht instrumentelle Kalküle, sondern das Bedürfnis nach Selbstachtung. Vormoderne Gesellschaften basieren auf einer institutionell verankerten, letztlich religiös begründeten Form kollektiver Identität. In modernen Gesellschaften dagegen gibt es, wie argumentiert wird, eine solche übergreifende kollektive Identität nicht mehr. An ihrer Stelle sei der Konsum zu einem »Medium der Vergesellschaftung " ${ }^{11}$ geworden; ähnlich sieht McCracken im Konsum »one of the ways in which order is created and maintained «. ${ }^{12}$ Der moderne Erlebniskonsum wirkt identitätsbildend, indem er jenseits der Anonymität des Marktes die Formierung sozialer Milieus und milieu-

8 Tanner 2009, S. 337.

9 McKendrick et al. 1982.

10 Schrage 2009, S. $103 \mathrm{f}$.

11 Ebd., S. 8.

12 McCracken 1988, S. xi. 
typischer Lebensstile vermittelt sowie personal strukturierte Anerkennungsverhältnisse ermöglicht. ${ }^{13}$ Die Konsumenten suchen die Befriedigung nicht nur materieller, sondern auch ästhetischer, moralischer und sogar spiritueller Bedürfnisse - Bedürfnisse, die in vergangenen Zeiten an die Religion adressiert wurden. Es gibt deshalb Autoren, die vom Konsum als einer »Ersatzreligion « sprechen, so Norbert Bolz ${ }^{14}$ in seinem unmittelbar nach dem 11. September 2001 geschriebenen »Konsumistischen Manifest «: »Nicht die Kirchen, sondern die Konsumtempel sind der Ort moderner Religiosität. [...] Das Ideal des Marketings ist die religiöse Ikonenverehrung. Heute kehren die Warenhäuser wieder an ihren Ursprung zurück. Die Pariser Passagen waren die ersten Kathedralen des Konsums. Und die Einkaufszentren der Gegenwart verwandeln sich in Schauplätze einer Wiederverzauberung der Welt, nach der wir uns gerade deshalb sehnen, weil jede Spur von Magie, Charisma und Zauber aus unserem aufgeklärten Alltag getilgt ist «.${ }^{15}$ Auch Jochen Hirschle vertritt die These, dass der Konsum die Funktion eines Religionsersatzes angenommen habe. Er versucht, sie auch durch eine eigene empirische Untersuchung zu belegen, deren zentraler Befund in einer negativen Korrelation zwischen der Häufigkeit von Gottesdienstbesuchen und konsumbezogenen Freizeitaktivitäten besteht. ${ }^{16}$

Es sind freilich, wie Bolz betont, wichtige Unterschiede zwischen den Identitätskonstruktionen der Religionen und denen des Konsums zu beachten: Im Gegensatz zu religiösen Identitäten sind Konsumidentitäten weder sozial ausschließender Art (man kann mehreren Konsummilieus angehören), noch gelten sie dauerhaft. Die Inklusions-/Exlusionsautomatik religiöser Identitäten mit ihren sowohl integrierenden als auch disruptiven Wirkungen wird vermieden. Konsum integriert die Gesellschaft auf eine zwar schwache und unbeständige, dafür aber friedliche Weise. Er stellt ein globales, die Grenzen von Nationen und Zivilisationen überschreitendes bzw. unterlaufendes Identifikationsangebot bereit, das zudem gerade durch seine Dynamik integriert: Die Konsumenten werden durch ihre individuellen Profilierungs- und Abgrenzungsbestrebungen gegen die herrschenden Trends gerade nicht exkludiert, sondern inkludiert. Bolz erblickt in dem konsumistischen Identitätsangebot die entscheidende Stärke der westlichen Zivilisation gegen politische und religiöse Fundamentalismen.

Interessanterweise ist das Verhältnis zwischen Konsumismus und Religion ein Thema auch in der Religionssoziologie. In der neueren religionssoziologischen Forschung wird eine Annäherung religiöser Deutungsangebote an die Welt des kommerziellen Konsums diagnostiziert. Das gilt, wie Hubert Knoblauch ${ }^{17}$ zeigt, nicht nur für die sogenannten »neuen « oder » populären « Religionen wie New Age, Esoterik oder Okkultismus, deren Angebote sich von dem kommerziellen WellnessMarkt oft kaum mehr unterscheiden. Auch die etablierten Kirchen suchen die Reso-

13 Zick-Varul 2004.

14 Bolz 2002, S. 98.

15 Ebd., S. 115 f.

16 Hirschle $2012 \mathrm{a}, \mathrm{b}$.

17 Knoblauch 2009. 
nanz auf ihre Verkündigung zu steigern, indem sie z. B. auf Elemente der Popkultur zurückgreifen. Während Konsumsoziologen eine Moralisierung, Ästhetisierung und Spiritualisierung des Konsums beobachten, sprechen Religionssoziologen von einem Trend zur Kommerzialisierung der Religion. Mit der Pluralisierung der Religionen und der Subjektivierung des religiösen Glaubens hat sich - so lautet der von Thomas Luckmann ${ }^{18}$ schon in den 1960er Jahren formulierte Befund - ein »Markt « der Religionen entwickelt, der auch die etablierten Bekenntnisse zu Anpassungen zwingt. Indem sie sich in der Konkurrenz gegeneinander profilieren und um »Kunden « werben müssen, bringen sie ihre Glaubensangebote unwillkürlich immer stärker in ein konsumaffines Format. Die Folge ist, wie Bryan Turner feststellt, dass »all forms of religion are now overlaid with consumerism and that many forms of religion have been commodified $\ll .{ }^{19}$ Mit der Privatisierung und Spiritualisierung des religiösen Glaubens erodiert, wie Turner argumentiert, die für das religiöse Weltbild konstitutive Dichotomie zwischen Transzendenz und Immanenz. Das »Jenseits « der Religionen und die Unendlichkeit der Optionen der Konsumwelt nähern sich bis zur Unkenntlichkeit an. In diesem Sinne findet die These von der sozialen Integrationsfunktion des Konsums Unterstützung auch von religionssoziologischer Seite.

(b) Eine dezidierte Gegenposition zur These von der sozialen Integrationsfunktion des Konsums hat Daniel Bell ${ }^{20}$ vertreten. Bei ihm ist der Konsumhedonismus gerade eine desintegrative Kraft, die im Widerspruch zur Logik der Produktionssphäre steht und die asketische Produktionsethik des Kapitalismus zersetzt. In seiner pessimistisch getönten Zeitdiagnose sieht Bell einen sich zuspitzenden Konflikt zwischen der für die kapitalistische Ökonomie konstitutiven protestantischen Ethik und den hedonistischen Idolen der modernen Kultur und Konsumwelt. Die moderne Kultur habe das Selbst vergöttert und führe zu einem hemmungslosen Ausleben hedonistischer Impulse. Die Folge sei eine wachsende Beliebigkeit des sozialen Verhaltens, die die für die kapitalistische Produktionsweise unentbehrliche Leistungsethik und Kooperationsdisziplin untergräbt. »In the world of capitalist enterprise ", so formuliert Bell seine These, »the nominal ethos in the spheres of production and organization is still one of work, delayed gratification, career orientation, devotion to the enterprise. Yet, on the marketing side, the sale of goods, packaged in the glossy images of glamour and sex, promotes a hedonistic way of life whose promise is the voluptuous gratification of the lineaments of desire. The consequence of this contradiction, as I put it in these pages, is that a corporation finds its people being straight by day and swinging by night $«{ }^{21}$ Letztlich sieht Bell keinen anderen Ausweg aus diesem Konflikt als einen höchst »traditionellen«: die Rückkehr zur traditionellen Religion.

Im Folgenden werde ich argumentieren, dass eine stichhaltige Begründung der These von der sozialintegrativen Funktion des modernen Konsums bis heute in der

18 Luckmann 1993.

19 Turner 2011, S. xii

20 Bell 1978.

21 Ebd, S. XXV.

Leviathan, 40. Jg., 4/2012 
Tat nicht vorliegt. Das gilt nicht nur für die oben referierten neueren konsum- und religionssoziologischen Ansätze, sondern auch für die ältere marxistische Theorie der Konsumästhetik, die diese These ebenfalls in einem allerdings ganz anderen Sinn schon vertreten hatte. Sie ist, wie ich zeigen möchte, ebenso wenig in der Lage, ihre Erklärungsansprüche einzulösen. Daraus folgt freilich keine Zustimmung zu dem Bell'schen Kulturpessimismus. Ich werde einen alternativen Ansatz skizzieren, mit dem sich - wie ich hoffe zeigen zu können - die These der sozialintegrativen Funktionen der Konsumdynamik gleichwohl rechtfertigen lässt. Aufbauend auf diesem Ansatz, werde ich eine elementare Typologie jener Funktionen entwickeln. Die Typologie konzentriert sich, um es zu betonen, auf die dynamischen Aspekte des Konsums; es wird somit keine umfassende Erklärung des Konsumhandelns beabsichtigt.

\section{Postmoderner Konsum und marxistische Analysen der Warenästhetik}

Die gegenwärtige Konsumforschung konzentriert sich auf die interpretative Rekonstruktion der "Erlebnisse ", die die heutige, oft als " postmodern « bezeichnete Konsumkultur den Kunden bietet. Konsumgüter und Dienstleistungen sollen nicht mehr nur physische Bedürfnisse befriedigen, sondern in dem Konsumenten Träume, Imaginationen, Phantasien auslösen. ${ }^{22}$ Diese Phantasien umfassen das gesamte Spektrum menschlicher Erfahrung, sie können ästhetischer, aber auch spiritueller und moralischer Art sein. Der Gebrauchswert der Dinge erfährt so eine symbolische Überhöhung; wichtig ist den Konsumenten nicht nur, was ein Artefakt konkret leistet, sondern was es leisten könnte, welche Lebensmöglichkeiten es vermittelt; ein Geländewagen etwa assoziiert die Vision eines Abenteurerlebens mitten in wilder Natur. ${ }^{23}$ Was die Konsumenten wollen, ist nicht eigentlich das Produkt, sondern die mit ihm verknüpften »Erlebnisse«. Die Wertbestimmung der Güter gewinnt damit eine »imaginäre " Dimension. ${ }^{24}$ Die heutige empirische Konsumsoziologie arbeitet daran, derartige Imaginationen klassifizierend zu erfassen und ihre Verdichtung zu Milieus und Lebensstilen zu beschreiben. ${ }^{25}$

Es bleibt jedoch die von Bell gestellte Frage, ob »Erlebnisse « allein sozialen Zusammenhalt stiften können. Was die Konsumsoziologie bei ihrer Fixierung auf die Erlebnisseite des Konsums ausblendet, ist der soziale Kontext des Handelns der Konsumenten, die konkrete Situation des Konsums, zu der auch immer der Zusammenhang der Konsumentenrolle mit den anderen von den beteiligten Personen wahrgenommenen Rollen gehört, insbesondere mit Arbeitsrollen. Jenseits der Tagträume der Konsumenten handelt es sich bei dem Konsum um eine reale Austauschbeziehung im Kontext umfassender, durch die Formen von Ware und Geld vermittelter sozialer Interdependenz. Wer konsumiert, erwartet, bedient zu werden: Das

22 Baudrillard 1998; Campbell 1987; Featherstone 2007.

23 Ullrich 2006.

24 Beckert 2011.

25 Vgl. exemplarisch Schulze 1993. 
Seil beim Bungee-Jumping muss zuverlässig befestigt sein, das Personal muss bei der Kreuzfahrt zu Diensten stehen, die Logistik bei der Trekking-Tour oder beim Versand über das Internet bestellter Produkte muss funktionieren. Die Mühe der Bedienung oft launischer und anspruchsvoller Kunden ist die unvermeidliche Negativseite des Konsumhedonismus. Um bei den Anbietern die Bereitschaft zu dieser Mühe zu wecken, müssen die Konsumenten in die Rolle der Kunden schlüpfen, und als solche brauchen sie Geld. Geld ist unentbehrlich nicht nur als Motivationsmittel auf der Leistungsseite, sondern ist auch konstitutiv für das Konsumerlebnis selbst. Der moderne Konsum ist wesentlich geldvermittelter Konsum; erlebnisträchtig ist nur, was gegen Geldzahlungen zu haben ist. Das kostenfreie romantische Wandern in freier Natur stellt kein »Erlebnis « im Sinne des Konsumhedonismus dar. Daher rührt auch das von Wolfgang Ullrich ${ }^{26}$ erwähnte »Konkurrenzverhältnis « zwischen Gütern und Geld. Denn Geld vermittelt Erlebnisse, selbst wenn man es nicht ausgibt, es stellt, wie Georg Simmel ${ }^{27}$ gezeigt hat, in gewisser Weise den Grund der mit dem Konsum verknüpften Wertempfindungen selbst dar und kann sie daher zumindest potenziell substituieren. Erst die Verfügung über Geld lässt jenen »mittleren Zustand « zwischen Haben und Nichthaben entstehen, in dem die Dinge uns überhaupt begehrenswert erscheinen. Der moderne Erlebniskonsum ist ein Begehren nach dem Begehren selbst. Sein Kern ist das Bedürfnis nach Geld, das der Kunde entweder schon haben oder sich durch Kredit beschaffen muss. Das bedeutet eine positive oder negative Festlegung seiner gesamten Lebenssituation. Eine Konsumsoziologie, die sich für die sozial integrativen Funktionen des Konsums interessiert, wird von diesen Randbedingungen nicht gut abstrahieren können. Aber das Geld, der Markt und die Produktion als Voraussetzungen des Konsums kommen in der auf die Erlebniswelt der Akteure fixierten ethnografischen Konsumforschung meist gar nicht vor bzw. werden als unproblematische Prämissen betrachtet.

Das war allerdings nicht immer so. Die - heute freilich fast in Vergessenheit geratene-Marx'sche und marxistisch orientierte Konsumkritik (Lukacs, Adorno/Horkheimer, Marcuse, Haug, Debord) hatte die strukturellen Bedingungen kapitalistischer Produktion sogar in das Zentrum ihrer Analysen gestellt, freilich um den Preis einer spiegelbildlichen, die Perspektive der Konsumenten weitgehend vernachlässigenden Vereinseitigung. Konsumgüter - so lautet die bekannte, auf Karl Marx zurückgehende Argumentation - haben im Kapitalismus nicht nur den Charakter von Gebrauchswerten, sondern von Waren, das heißt Tauschwerten. Der Kapitalismus folgt einer Logik der Maximierung von Mehrwert und Profit, nicht der optimalen Befriedigung von Bedürfnissen. Das den Tausch scheinbar nur Vermittelnde - das Geld als Ausdruck abstrakter Arbeit - wird zum dominanten Ziel und Zweck der Reproduktion. Um den Mehrwert realisieren zu können, muss auch die Nachfrage permanent steigen, und zwar auch dann, wenn die Bedürfnisse der Konsumenten » eigentlich « gesättigt sind. Die Waren werfen daher, wie Marx es formuliert hatte, "Liebesaugen " nach dem Geld der potenziellen Käufer; ihre Anbieter versuchen, die Begehrlichkeit der Kunden zu wecken. Es kommt zu einer Funktionali-

26 Ullrich 2006, S. 59.

27 Simmel 1989 [1900].

Leviathan, 40. Jg., 4/2012 
sierung des Gebrauchswerts und mit ihm der Bedürfnisse der Konsumenten durch den Tauschwert. Um die Mehrwertproduktion in Gang zu halten, muss die Kaufbereitschaft der Konsumenten durch Werbung, Verpackung, immer neue Moden stimuliert werden. Im Monopolkapitalismus nehmen die Konzerne, wie Adorno und Horkheimer ${ }^{28}$ in ihrer Kritik der Kulturindustrie ausgeführt hatten, das Unbewusste der Konsumenten planmäßig mit ihren Marketing-Strategien in Regie. Die Konsumund Kulturindustrie stellte sich ihnen als ein System umfassender, mit technologischen Mitteln ausgeübter Herrschaft der Industrie über die Konsumenten dar. Diese Einschätzung war damals keineswegs aus der Luft gegriffen und ist es auch heute nicht. In seiner zum Bestseller avancierten Studie über die »Hidden Persuaders" hatte Vance Packard ${ }^{29}$ die im Konsummarketing angewandten Methoden psychologischer Motivanalyse analysiert. Er zeigte, wie diese Methoden darauf abzielten, das rationale Urteilsvermögen der Konsumenten zu umgehen und den Marktumsatz durch Mobilisierung unterbewusster Wünsche der Käufer zu steigern. ${ }^{30}$ Ähnlich hatte John K. Galbraith ${ }^{31}$ den Konsumenten als Marionette auf Umsatzwachstum und "planned obsolescence " gerichteter Marketing-Strategien der Konzerne gesehen. Die Konsumdynamik erscheint aus dieser Sicht nicht als Ausdruck der »Souveränität« der Konsumenten, sondern gerade ihrer Heteronomie. Der Konsum und seine Ästhetik werden hier zwar als Medium sozialer Integration der Gesellschaft gesehen - jedoch einer »falschen ", entfremdeten, durch irrationale Kräfte bestimmten Integration, die Produzenten wie Konsumenten gleichermaßen unterdrückt. Der Konsumkritik wird damit die Aufgabe politischer und gesellschaftlicher Aufklärung zugewiesen.

Heute haben diese Verführungs- und Manipulationstheorien des Konsums deutlich an Einfluss verloren, sind jedoch keineswegs verschwunden. Einflussreiche Beispiele sind Naomi Kleins "No Logo « 32 oder Benjamin Barbers Kritik an den infantilisierenden Mechanismen des Konsums. ${ }^{33}$ So sehr man den Botschaften dieser Autoren in manchen Punkten zustimmen möchte, so unabweisbar ist die Kritik an dem ihnen zugrundeliegenden Denkansatz. Nur zwei Punkte der einschlägigen Kritik möchte ich hervorheben: Erstens: Problematisch ist die funktionalistische Schlagseite der Manipulationstheorien. Gesellschaftliche Integration wird als prinzipiell selbsttätiger, durch anonyme Strukturgesetzlichkeiten bestimmter Prozess verstanden, in dem den Akteuren nur die Rolle ausführender Organe zukommt. Die aktive Rolle individuellen Handelns, und das heißt methodisch: die Notwendigkeit

28 Adorno, Horkheimer 1969 [1944].

29 Packard 1957.

30 Ein Autohändler habe, wie Packard berichtet, die Jagd nach wirksameren Verkaufsmethoden »sehr bildhaft in die Worte zusammengefaßt: >Wenn die Kauflust noch weiter nachläßt, werden wir dem Verbraucher eins über den Kopf geben und ihn den Kaufvertrag unterschreiben lassen müssen, solange er bewußtlos (unconscious) ist« (ebd., S. 19).

31 Galbraith 1958.

32 Klein 2001.

33 Barber 2007. 
einer eigenen Analyseebene zur empirischen Erfassung dieses Handelns, wird vernachlässigt. Es muss daran erinnert werden, dass es immer die Konsumenten selbst sind, die über den Kauf entscheiden, auch wenn ihre Entscheidungen stets sozial kontextuiert und zweifellos nur begrenzt rational sind. Zweitens: Die Manipulationstheorien leben von unausgewiesenen, letztlich normativ begründeten Annahmen über die "Vernünftigkeit " von Bedürfnissen und die Art eines »selbstbestimmten " Handelns der Konsumenten. Diese Annahmen reichen von den revolutionstheoretischen Spekulationen bei Georg Lukacs, der Randgruppentheorie bei Herbert Marcuse bis hin - in jüngster Zeit - zu Axel Honneths ${ }^{34}$ Interpretation von Konsumgenossenschaften als authentischer Form reziproker "Anerkennung " in der Konsumsphäre. Aber es handelt sich hier durchweg um Aussagen darüber, was die Konsumenten nach Meinung der Autoren tun sollten. Die Aufgabe wissenschaftlicher Rekonstruktion sollte jedoch darin bestehen, das gesamte Spektrum der Motive der Konsumenten unabhängig von theoretischen Voreinstellungen bezüglich der geschichtlichen Mission des Proletariats oder der moralischen Strukturierung von Konsummärkten zu erfassen. Sieht man von Mangelsituationen ab, wie sie in Europa in der ersten Hälfte des 20. Jahrhunderts zeitweilig herrschten, wird man nüchtern konstatieren müssen, dass die empirische Evidenz für ein »solidarisches " Handeln der Konsumenten nicht eben überwältigend ist. Auch mit den aktuell vieldiskutierten Trends zur »Ethisierung « und »Moralisierung « des Konsums, ${ }^{35}$ die sich nur auf bestimmte Käuferschichten beschränken und selbst eher eine Form imaginativen Konsums darstellen, lässt sich die These der Konsumentensolidarität kaum überzeugend belegen. Viel mehr spricht dafür, dass die Konsumenten mit wachsendem Einkommen zunehmend an individueller »Souveränität « und weniger an »Solidarität « interessiert sind. Sie möchten die Fülle der mit dem Geld verknüpften Wahlmöglichkeiten nutzen und ihre persönlichen Wünsche ausleben. Kann man solche Wünsche von vornherein als amoralisch disqualifizieren? Damit sind wir wieder bei dem heute vorherrschenden » postmodernen «, erlebniszentrierten Ansatz angelangt. Mit anderen Worten: Wir sind so klug wie zuvor.

\section{Ein Mehrebenenmodell der Konsumdynamik}

Gibt es eine Möglichkeit, aus diesem fehlerhaften Zirkel herauszukommen? Dazu müsste es gelingen, die disjecta membra der Soziologie des Konsums zusammenzuführen, das heißt: die Konsumentenrolle in ihrem Zusammenhang mit der Produzentenrolle zu analysieren, ohne das eine dem anderen zu subsumieren; die Strukturbedingungen der Konsumdynamik zu erfassen, ohne dabei die Autonomie der Akteure zu vernachlässigen. Dass dieses keineswegs unmöglich ist, möchte ich im dritten und letzten Teil meiner Überlegungen zeigen. Um Anregungen für die Entwicklung einer differenzierten, weder produktivistisch/modern noch konsumistisch/ postmodern vereinseitigen Konsumsoziologie zu bekommen, ist zunächst der Rück-

34 Honneth 2011, S. $360 \mathrm{f}$.

35 Stehr 2007; Zick-Varul 2009.

Leviathan, 40. Jg., 4/2012 
griff auf aktuelle Entwicklungen der soziologischen Theorie und Methodologie hilfreich. Ein vielversprechendes Angebot dazu scheint mir das auf Coleman, Lindenberg und Esser zurückgehende Konzept der »soziologischen Erklärung « zu sein. Dabei muss man, wie ich sogleich hinzufügen möchte, die rational-choice-theoretische Schlagseite der handlungstheoretischen Konzeptualisierungen Colemans und Essers nicht teilen; auch ich werde das, Mayntz und Scharpf ${ }^{36}$ folgend, nicht tun. Die methodologischen Grundforderungen an eine tragfähige soziologische Erklärung, die sich aus dem Konzept ergibt, hat schon Coleman in seinem berühmten »Badewannenmodell « zusammengefasst, das heute weitgehend Gemeingut geworden ist; ich erinnere deshalb hier nur kurz daran. Eine soziologische Erklärung besteht danach im Prinzip aus drei Schritten: ${ }^{37}$ Der erste Schritt ist die Rekonstruktion der sozialen Situation, die Gegenstand der Untersuchung ist, und zwar einmal der Situation, wie sie sich dem wissenschaftlichen Beobachter, und zum anderen, wie sie sich den Akteuren selbst darstellt. Diese beiden Perspektiven müssen durch »Brückenhypothesen « miteinander verbunden werden (» Logik der Situation « nach Esser). Der zweite Schritt ist die theoretisch begründete Erklärung des Handelns der Individuen in der gegebenen Situation (»Logik der Selektion «), der dritte die Ableitung der neu konstituierten kollektiven Situation aufgrund der aggregierten Effekte der individuellen Handlungen (»Logik der Aggregation «). Entscheidend ist immer der Wechsel von der Makro- auf die Mikroebene und zurück, wobei die Analysezyklen wiederholt, verkettet und so für die Untersuchung dynamischer Prozesse fruchtbar gemacht werden können.

Mir scheint es vielversprechend zu sein, diesen Ansatz auch bei der Analyse der Konsumdynamik und ihrer sozialen Integrationsfunktionen $\mathrm{zu}$ berücksichtigen. Glücklicherweise muss auch hier keineswegs völliges Neuland betreten werden, denn innerhalb der Konsumsoziologie liegen theoretische Ansätze zur Erklärung der Konsumdynamik durchaus vor, die den Mehrebenencharakter des Konsumhandelns im Sinne des genannten Modells berücksichtigen (auch wenn sie sich nicht explizit darauf beziehen). Einschlägig ist hier zunächst der bekannte und in der Konsumsoziologie bis heute einflussreiche Ansatz Bourdieus. ${ }^{38}$ Er konzentriert sich allerdings auf die Analyse des Zusammenhangs zwischen Konsummustern und sozialen Lagen und die Klärung der das soziale Feld des Konsums bestimmenden Machtverhältnisse. Bourdieus Untersuchung erfasst soziale Chancenverteilungen und deren Determinanten zu einem gegebenen Zeitpunkt und ist insofern eher "statisch « angelegt. Die Bestimmungsgründe der Dynamik des Konsums und der Beitrag, den die Konsumdynamik für die kapitalistische Dynamik insgesamt leistet, stehen nicht im Zentrum der Bourdieu'schen Perspektive. Für die Klärung der dynamischen Seiten des Konsums scheint es mir produktiver, am klassischen, bereits von Georg Simmel ${ }^{39}$ formulierten »trickle down «-Ansatz auzuknüpfen, der von man-

36 Mayntz, Scharpf 1995.

37 Esser 1993; Esser 1999.

38 Bourdieu 1982.

39 Vgl. Simmel 1989 [1900], S. 639, 649; Simmel 1995 [1905]. 
chen Konsumforschern zwar als veraltet betrachtet wird, gegen diese Kritik jedoch (meiner Meinung nach mit Recht) durch McCracken in Schutz genommen wurde. ${ }^{40}$

Zentral für diesen Ansatz ist der Gedanke, dass die Konsumdynamik im Zusammenhang mit Prozessen sozialer Mobilität analysiert werden muss. Soziale Aufsteiger bemühen sich, ihre pekuniäre Potenz auch äußerlich sichtbar in soziale Respektabilität zu übersetzen. Dazu ahmen sie die Lebensstile der jeweils höheren Schichten nach und eignen sich deren Konsumsymbole an. Diese diffundieren damit gleichsam nach »unten « (trickle down) und verlieren ihren Distinktionswert. Die höheren Schichten reagieren darauf, indem sie ihre alten Symbole aufgeben und neue Vorlieben entwickeln, durch die sie sich wiederum von der Masse abheben können. Angetrieben durch die gegenläufigen Motive der Imitation einerseits, der Distinktion andererseits, kommt so ein sich selbst perpetuierender Mechanismus der Erzeugung immer neuer Konsummoden in Gang. Dabei werden die Eliten allein durch das Motiv der Distinktion getrieben, die unteren Schichten dagegen durch das der Imitation. In den mittleren Schichten herrschen dagegen ambivalente, zwischen Imitation und Distinktion hin- und herschwankende Motivlagen vor. ${ }^{41}$

Der »trickle down «-Ansatz in seiner neueren, vor allem durch McCracken formulierten Fassung berücksichtigt in mehrfacher Hinsicht die oben formulierten Forderungen an eine nichtreduktionistische Theorie der Konsumdynamik: Er berücksichtigt den Mehrebenencharakter des Handelns, indem er systematisch zwischen der durch strukturelle soziale Ungleichheit geprägten sozialen Situation der Konsumenten und der Reaktion der Konsumenten auf diese Situation unterscheidet. Darüber hinaus berücksichtigt er die ungeplanten Aggregatwirkungen dieses Handelns und die erneute Reaktion der Akteure auf diese Aggregatwirkungen. Damit lässt sich auch die häufig als Gegenmodell betrachtete, auf Colin Campbell ${ }^{42}$ zurückgehende Theorie des »imaginären Konsums « zu einem guten Teil mit dem »trickle down «-Ansatz verknüpfen, denn das erneute Differenzierungsverhalten der oberen Schichten lässt sich als Form »imaginären « Konsums im Sinne Campbells interpretieren. Ein weiterer Vorteil des Ansatzes ist, dass die Verengung der Analyse auf die Erlebnisdimension vermieden wird und das Konsumverhalten im Kontext der durch Auf- oder Abstiegsprozesse geprägten umfassenderen Situation der Konsumenten betrachtet wird.

Soziale Auf- und Abstiegsprozesse setzen eine strukturell durch vertikale Ungleichheit bestimmte soziale Situation voraus. Moderne, kapitalistische Gesellschaften sind dadurch charakterisiert, dass ältere, ständische Strukturen sozialer Ungleichheit durch die moderne, durch Besitz bzw. Nichtbesitz von Kapital bestimmte Dichotomie der Klassen überlagert und dominiert wird. Im Gegensatz zu den sozial relativ geschlossenen ständischen Ungleichheiten ist die Klassendichotomie formal offen. Individuelle Aufstiege aus den nichtbesitzenden in die besitzenden

40 McCracken 1985; McCracken 1988; vgl. auch McKendrick 1997; Brewer 1997.

41 McCracken 1985; McCracken 1988.

42 Campbell 1987.

Leviathan, 40. Jg., 4/2012 
Klassen sind unabhängig von der persönlichen Herkunft prinzipiell möglich, wenn auch faktisch eher selten; ebenso sind Abstiege aus den besitzenden Klassen möglich. Die formale Offenheit der Klassenstruktur kann trotz der faktisch geringen Mobilitätschancen als mächtiges Aufstiegsmotiv für Individuen aus den besitzlosen Schichten wirken. Dabei scheinen die Aufstiegsmotivation und zugleich die positive Wahrnehmung der Aufstiegschancen in den unteren, sogenannten »kleinbürgerlichen « Mittelschichten besonders ausgeprägt zu sein. In kapitalistischen Gesellschaften gibt es einen quasi "außerinstitutionellen « Weg des Aufstiegs jenseits formalisierter Karrieren und Qualifikationshierarchien über den Erfolg am Markt. Das Spezifikum dieser Aufstiegsform ist, dass sie sich nicht nur im Rahmen gegebener sozioökonomischer Strukturen vollzieht, sondern mittels Veränderungen dieser Strukturen selbst. Die Konsumdynamik muss - das ist die These, die ich aus dem »trickle down «-Konzept ableiten möchte - zum einen als Moment derartiger Aufstiegsprozesse und -ambitionen analysiert werden (a). Zum anderen stellt sie eine Reaktion auf die ungeplanten Aggregatwirkungen dar, die durch solche positiven Mobilitätsprozesse hervorgerufen werden (b). Beide Möglichkeiten sollen im Folgenden genauer betrachtet werden:

(a) Der Erfolg von Aufsteigern am Markt setzt, wie bereits Schumpeter und Merton gezeigt haben, "innovative " individuelle Anstrengungen im weitesten Sinne voraus, die sich sowohl auf die Produktions- als auch auf die Konsumsphäre beziehen. Das Handeln unternehmerischer Aufsteiger richtet sich auf die Transzendierung ihrer gegebenen Lebens- und Reproduktionsformen - derart, dass nicht nur die Position des Aufsteigers in einem gegebenen sozioökonomischen Koordinatensystem sich verändert, sondern die Struktur des Systems selbst. ${ }^{43}$ Innovatives produktives Handeln kann die unterschiedlichsten Formen annehmen: Entdeckung und Ausbau von »Marktnischen ", Entwicklung und Vermarktung neuer Technologien, Geschäftsmodelle oder Produkte. All das setzt Lernbereitschaft und die Entwicklung neuer persönlicher Qualifikationen voraus. Um ihr soziales »Kapital« zu erweitern, ist es für unternehmerische Aufsteiger wichtig, sich durch eine innovative Selbstdarstellung auch auf der Ebene des Konsums zu profilieren. »Innovation « ist ein Prozess, der sich sowohl auf der Ebene der Arbeit wie der des Konsums abspielt. Arbeit und Konsum können freilich auf sehr unterschiedliche Weise lebensgeschichtlich sequenziert werden. Idealtypisch lassen sich zwei Extremvarianten unterscheiden.

Die erste Variante möchte ich als das Modell des viktorianischen Aufstiegs bezeichnen, der durch das Motto bestimmt wird: Erst arbeiten und sparen, dann konsumieren. Konstitutiv für diesen bei kleinbürgerlichen Unternehmern und Freiberuflern verbreiteten Typus ist eine asketische Arbeitshaltung, die eine hohe Leistungsbereitschaft, Frustrationstoleranz und Fähigkeit zu aufgeschobener Bedürfnisbefriedigung einschließt. Jürgen Kocka beschreibt diesen Habitus, der ihm zufolge bei den deutschen Unternehmern in der Zeit der frühen Industrialisierung eine wichtige Rolle spielte, wie folgt: »Arbeitserfolg sicherte nicht nur die Versorgung der eigenen Familie, er begründete auch den Stolz auf die eigene Leistung, die 
individuelle persönliche Ehre, die nach den bürgerlichen Vorstellungen jener Zeit untrennbar mit Rechtlichkeit, Qualität, Solidarität und Fleiß verknüpft war; er war für die häufig sehr religiösen, vor allem protestantischen Unternehmer jener Zeit zugleich Unterpfand ihrer Auserwähltheit durch Gott. Dieser Begriff von Arbeit legitimierte den Fleiß und die Sparsamkeit auch im Privatleben, den Ordnungssinn und die Nüchternheit, die Zweckrationalität und die Freude am Gelderwerb «. ${ }^{44}$ Die asketische Grundeinstellung blieb, wie Kocka hinzufügt, häufig auch dort erhalten, wo die religiöse Motivation keine Rolle mehr spielte. Der Konsum bildet für die diesem Typus zuzurechnenden Personen über weite Strecken ihrer Karriere hinweg eigentlich gar kein Thema. Erst in Phasen weit fortgeschrittener Konsolidierung des geschäftlichen Erfolgs kann er ins Spiel kommen und hat dann die Funktion, den Erfolg sozial sichtbar zu machen. Der Konsum soll den durch den Markterfolg faktisch und latent schon erreichten sozialen Statusgewinn auch äußerlich zum Ausdruck bringen. Er hat hier - in anderen Worten - die soziale Funktion der Anerkennung; das heißt die Aufsteiger beanspruchen Mitgliedschaft in der neuen, höheren Statusgruppe und möchten von deren Mitgliedern als ihresgleichen anerkannt werden. Die Orientierung an den ästhetischen Idolen der Zielgruppe spielt dann eine zentrale Rolle; der Konsum ist also in erster Linie Positionskonsum.

Die zweite Variante möchte ich als das Modell des konsumistischen Aufstiegs bezeichnen. Die lebensgeschichtliche Sequenzierung von Arbeit und Konsum verläuft hier umgekehrt wie im Modell des viktorianischen Aufstiegs und gehorcht dem Motto: Erst konsumieren, dann hart arbeiten und die aufgenommenen Schulden zurückzahlen. Es wird hier gleichsam »vorwegnehmend « konsumiert und dafür Kredit aufgenommen nach dem Prinzip: Mehr scheinen als sein. Das »dicke Ende « kommt dann in Form von Zahlungsverpflichtungen hinterher, die durch Sparsamkeit und harte Arbeit eingelöst werden müssen. Wie Lendol Calder in seiner historischen Studie über die Finanzierung des amerikanischen Traums ${ }^{45}$ gezeigt hat, spielte dieser Typus in der amerikanischen Wirtschaftsgeschichte eine zentrale Rolle (in Europa, jedenfalls auf dem europäischen Kontinent, scheint seine Bedeutung dagegen geringer zu sein). Bereits vor dem Ersten Weltkrieg waren Konsumentenund Ratenkredite in den amerikanischen Mittelschichten und unteren Mittelschichten stark verbreitet; die Anschaffung von dauerhaften Gebrauchsgütern wie Nähmaschinen, Klaviere, Automobile wurde in breitem Umfang »auf Pump « finanziert. Zugleich kam es zu einer gesellschaftlichen Legitimierung des Konsumkredits durch entsprechende Narrative, bei deren Entstehung sowohl Wohltätigkeitsorganisationen (etwa die Russell Sage Foundation) als auch ökonomische Experten eine wichtige Rolle spielten. ${ }^{46}$ Die niedrige Ausfallrate der Konsumentenkredite deutete zumindest in der untersuchten Zeit bis zum Zweiten Weltkrieg - auf eine hohe Arbeits- und Zahlungsmoral der Konsumenten hin. Der Konsumkredit wirkt hier

44 Kocka 1975, S. 58.

45 Calder 1999.

46 Calder erwähnt in diesem Zusammenhang unter anderem Edwin R. A. Seligman, der eine einflussreiche Untersuchung mit dem Titel »The economics of installment selling « veröffentlicht hatte; vgl. Seligman 1927. 
als eine Instanz, die die Wunschwelt des Konsums in einen Antrieb für produktive Askese verwandelt. Dies übersehen zu haben hält Calder Bell vor. ${ }^{47}$ Auf dem Umweg über den Kredit hat der Konsum hier - mit anderen Worten - die soziale Funktion der Disziplinierung. Die Konsumenten übertragen die Disziplinierung, zu der sie selbst unmittelbar nicht fähig sind, an den Kreditgeber als externe Instanz. Auch in diesem Fall hat der Konsum in erster Line den Charakter von Positionskonsum. Identifikationsobjekt ist aber nicht jene Statusgruppe, die man schon erreicht zu haben meint, sondern diejenige, die man erst erreichen möchte.

(b) Die Aggregatwirkungen viktorianischer oder konsumistischer Aufstiege lassen eine neue strukturelle Situation entstehen, die vor allem die Vermögenden vor neue Herausforderungen stellt. Wir betrachten jetzt die Lage nicht von Personen, die das für den Konsum nötige Geld nicht haben und sich leihen müssen, auch nicht von solchen, die das Geld durch eigene harte Arbeit erworben haben und erst dann damit beginnen, die mit ihm verknüpften Freiheiten zu erfahren. Es geht vielmehr nun um Personen, die es schlicht »haben ", das heißt für die der Besitz von Geld und (in der Regel ererbtem) Vermögen selbstverständlicher Hintergrund aller Lebensdispositionen ist. Wer über Geldvermögen verfügt, muss sich über die komplementäre Schuld keine Gedanken machen. Er/sie kann nicht nur die gewünschten Kaufobjekte, sondern auch die Tauschpartner frei wählen, er/sie kann sich aus den Zwängen der Zeit und denen des Raums befreien. Geld ist, wie bereits Simmel ${ }^{48}$ herausgearbeitet hatte, die Grundlage der modernen Ästhetisierung des Konsums, nicht nur im Sinne einer » externen « Voraussetzung oder Restriktion, sondern einer » inneren « Bedingung. Denn erst die hinreichende Verfügung über Geld schafft, wie oben schon angemerkt, jenen "mittleren Zustand zwischen Haben und Nichthaben" (Simmel), in dem die Dinge uns weder unerreichbar fern noch in unserem unmittelbaren Besitz sind und so einen »Wert « für uns überhaupt erst gewinnen können. $\mathrm{Zu}$ den durch das Geld gestifteten Freiheiten gehört auch, dass der Vermögende sie noch nicht einmal durch Konsum ausleben muss. Er kann, wie Simmel ${ }^{49}$ in seinem Portrait des »Geizigen « gezeigt hatte, einen asketischen Lebensstil pflegen und sich auf den Genuss der bloßen Möglichkeit des im Geld gespeicherten Lebens beschränken, indem er sein Vermögen akkumuliert.

Aber die Freiheit des Geldes wäre nichts, wenn sie nicht auch ausgelebt werden könnte und würde. Reichtum muss auch, wie Veblen und Bourdieu deutlich machten, sozial sichtbar gemacht werden: durch einen konsequent ästhetisierenden Konsum- und Lebensstil, der die soziale Funktion der Distinktion erfüllt. Der Reiche hat in seiner Lebensführung nichts anderes gelten zu lassen als den "guten Geschmack «, der freilich spezifischen historischen und kulturellen Konventionen unterliegt. Die Symbolik des Distinktionskonsums lässt sich zwar, wie die Kritiker der Theorie des Positionskonsums betonen, nicht unmittelbar einer Logik positionaler Konkurrenz subsumieren, sondern ist durch das Streben nach ästhetischer

47 Calder 1999, S. 299 f.

48 Simmel 1989 [1900].

49 Ebd., S. 305 f. 
Perfektion geprägt. Aber realiter kann dieses Streben nur unter der Prämisse des Vermögensbesitzes zum Tragen kommen. Ästhetische Kompromisslosigkeit im Konsum kann sich nur leisten, wer auf Geld keine Rücksicht zu nehmen braucht. Gerade der ästhetisch autonome Konsum impliziert insofern - dies hat Bourdieu mit Recht betont - ein sozial hierarchisierendes Element. Die gesellschaftliche Vorbildfunktion des Lebensstils der Vermögenden - dies ist gegen Esposito ${ }^{50}$ und Schrage ${ }^{51}$ einzuwenden - bleibt ungeachtet der Erosion ständischer Leitbilder gerade in fortgeschrittenen kapitalistischen Gesellschaften intakt. Er wird hier, wie Juliet Schor in ihren Analysen der aktuellen Entwicklungen des Massenkonsums in den USA gezeigt hat, durch den Einfluss der modernen Kommunikationsmedien noch verstärkt. Fernsehen und Internet tragen entscheidend dazu bei, die Konsumsymbole der Reichen zu Massenidolen zu erheben. ${ }^{52}$

Werden die Insignien des herrschenden "guten Geschmacks" progressiv durch soziale Aufsteiger angeeignet, so werden damit zwar noch nicht gleich alle Unterschiede eingeebnet. Denn die neureichen Aufsteiger sind, wie Bourdieu herausgearbeitet hat, noch immer an der Gezwungenheit oder auch an der Maßlosigkeit ihres Habitus erkennbar; es fehlt ihnen die lockere Selbstverständlichkeit der alten Eliten. Gleichwohl dürfte auf der Seite der Eliten das Abgrenzungsbedürfnis wachsen; die Folge ist eine Dynamisierung auch des Distinktionskonsums. Wie im »trickle down «-Modell gezeigt, reagieren die Reichen auf die Aneignung ihrer Symbole durch die Aufsteiger mit der Entwicklung neuer Symbole und Träume, die die herrschenden ästhetischen Konventionen durchbrechen. Maßstäbe setzen hier z. B. Modemessen, die die für die Eliten maßgeblichen Innovationen zeigen. Das kann auch in der Form geschehen, dass die nach oben gerichtete Nachahmungstendenz sozialer Aufsteiger von den Modemachern ironisch umgekehrt wird, etwa indem Konsumattribute der Unterschichten auf einmal für ästhetisch vorbildlich erklärt werden (»trickle up«statt »trickle down«). Diese Dynamik entspricht durchaus der von Campbell beschriebenen Logik des »imaginären « Konsums: Unter dem Einfluss der Werbung entwickeln die Konsumenten immer neue Tagträume und stoßen in neue "Erlebniswelten " vor. Aber ihr sozialer Effekt liegt in der Wiederherstellung der Distinktionswirkungen des Konsums. Mit Talcott Parsons könnte man auch formulieren, dass der Positionskonsum die Funktion der »pattern maintenance « erfüllt, indem er die Vorbildlichkeit der Lebensstile der Eliten markiert. Aber auch diese Funktion kann im modernen Kapitalismus nur durch Dynamisierung und Entgrenzung gewährleistet werden.

50 Esposito 2004.

51 Schrage 2009.

52 Schor 1998.

Leviathan, 40. Jg., 4/2012 


\section{Zusammenfassung}

Meine These kann ich so zusammenfassen, dass der moderne Erlebniskonsum ungeachtet seiner Modeabhängigkeit, Dynamik und seiner manchmal exzessiven Begleiterscheinungen als ein Faktor »moralischer Integration « der Gesellschaft betrachtet werden kann. Insoweit ist Bolz gegen Bell Recht zu geben, und zustimmen würde ich Bolz auch hinsichtlich seiner Charakterisierung der Weise, wie der moderne Konsum sozial integrierend wirkt. Mit der Art, wie sie sich kleiden, ernähren, wohnen usw., signalisieren die Individuen nicht nur Konformität mit dem herrschenden Geschmack und dessen klassen- bzw. schichtspezifischen Differenzierungen und bringen so ihre Konformität mit der sozialen Ordnung zum Ausdruck. Der moderne Konsum integriert vielmehr gerade durch seine Dynamik, das heißt durch die ihm innewohnende Tendenz, herrschende alltagsästhetische Konventionen in Frage zu stellen. Die paradoxe, sowohl inkludierende als auch exkludierende Logik sozialer Identitäten wird durch Dynamisierung unterlaufen, indem die Konsumenten auf einen nie endenden Weg zu sich selbst geschickt werden. Die Konsequenz ist die Unersättlichkeit des modernen Konsums, das durch die Marketing-Strategien der Industrie stimulierte Trachten der Konsumenten nach immer neuen »Erlebnissen «. Aber das ist der Preis, der für die Friedlichkeit dieser Form sozialer Integration zu zahlen ist.

Dass wir es hier mit einem Phänomen sozialer Integration und nicht etwa, wie Bell meint, mit einer kulturzerstörenden Überhöhung des Selbst zu tun haben, erschließt sich freilich erst, wenn man die Konsumentenrolle nicht länger isoliert analysiert, sondern ihrem realen Zusammenhang mit der Geld- bzw. Warenförmigkeit des Konsums sowie mit den Arbeitsrollen und sozialen Mobilitätsprozessen der Konsumenten Rechnung trägt. Konsum setzt individuelle Zahlungsfähigkeit voraus, und die Rekonstruktion der Bedingungen dieser Zahlungsfähigkeit sollte einen integralen Bestandteil der Analyse des Konsumentenhandelns bilden und nicht nur als dessen » externe " Voraussetzung betrachtet werden. Wie eine solche integrierte Analyse vorgenommen werden könnte, habe ich mit meiner Typologie zu zeigen versucht. Die sozialmoralischen Funktionen, die der Konsum erfüllen kann, lassen sich danach mit den Begriffen Anerkennung, Disziplinierung und Distinktion charakterisieren. Aus der von mir vorgeschlagenen Konzeptualisierung folgt schließlich, dass die sozialintegrativen Wirkungen des Konsums nicht so grenzenlos sind, wie Bolz es hinstellt. Denn vorausgesetzt werden, wie betont, Akteure, die in der Lage sind, ihre Kredit- und Zahlungsverpflichtungen zu erfüllen. Die sozial integrative Wirkung des Konsums findet ihre Grenze an der individuellen Zahlungsfähigkeit und der Fähigkeit der Regeneration dieser Zahlungsfähigkeit durch Arbeitseinkommen oder Kapitalbesitz. Zahlungsunfähigkeit bedeutet Exklusion. Schon der Arme, der immer nur das Billigste und Nötigste kaufen und nicht wählen kann, »konsumiert « nicht eigentlich und befindet sich in einer Lage latenter Exklusion. Erst recht kann dort, wo es zur Überschuldung und Zahlungsunfähigkeit privater Haushalte in massenhaftem Umfang kommt, wie in der Gegenwart, auch von einer integrativen Wirkung des Konsums keine Rede mehr sein. 


\section{Literatur}

Adorno, Theodor W.; Horkheimer, Max 1969 [1944]. Dialektik der Aufklärung. Frankfurt a. M.: Fischer.

Barber, Benjamin R. 2007. Consumed: how markets corrupt children, infantilize adults and swallow citizens as a whole. New York: Norton.

Baudrillard, Jean 1998. The consumer society. Myths and structures. London: Sage.

Beckert, Jens 2011. »The transcending power of goods. Imaginative value in the economy «, in The worth of goods. Valuation and pricing in the economy, hrsg. v. Beckert, Jens; Aspers, Patrik, S. 106-130. Oxford: Oxford University Press.

Bell, Daniel 1978. The cultural contradictions of capitalism. New York: Basic Books (deutsche Ausgabe: Bell, Daniel 1991. Die kulturellen Widersprüche des Kapitalismus. Frankfurt a. M.: Campus).

Bolz, Norbert 2002. Das konsumistische Manifest. München: Fink.

Bourdieu, Pierre 1982. Die feinen Unterschiede. Kritik der gesellschaftlichen Urteilskraft. Frankfurt a. M.: Suhrkamp.

Brewer, John 1997. »Was können wir aus der Geschichte der frühen Neuzeit für die moderne Konsumgeschichte lernen?", in Europäische Konsumgeschichte. Zur Gesellschafts- und Kulturgeschichte des Konsums (18.-20. Jahrhundert), hrsg. v. Siegrist, Hannes; Kaelble, Hartmut; Kocka, Jürgen, S. 51-74. Frankfurt a. M.: Campus.

Calder, Lendol 1999. Financing the American dream. A cultural history of the consumer credit. Princeton: Princeton University Press.

Campbell, Colin 1987. The romantic ethic and the spirit of modern consumerism. Milton Keynes: Blackwell.

Deutschmann, Christoph 2008. Kapitalistische Dynamik. Eine gesellschaftstheoretische Perspektive. Wiesbaden: VS Verlag für Sozialwissenschaften.

Deutschmann, Christoph 2009. Soziologie kapitalistischer Dynamik. MPIfG Working Paper 09/ 5. Köln: Max-Planck-Institut für Gesellschaftsforschung.

Deutschmann, Christoph 2011. "A pragmatist theory of capitalism «, in: Socio-Economic Review 9, 1, S. 83-106.

Douglas, Mary; Isherwood, Baron 1996 [1979]. The world of goods. Toward an anthropology of consumption. London: Routledge.

Esposito, Elena 2004. Die Verbindlichkeit des Vorübergehenden: Paradoxien der Mode. Frankfurt a. M.: Suhrkamp.

Esser, Hartmut 1993. Soziologie. Allgemeine Grundlagen. Frankfurt a. M.: Campus.

Esser, Hartmut 1999. Soziologie. Spezielle Grundlagen. Bd. 1: Situationslogik und Handeln. Frankfurt a. M.: Campus.

Featherstone, Mike 2007. Consumer culture and postmodernism. 2. Auflage. London: Sage.

Galbraith, John K. 1958. The affluent society. 2. Auflage. London: Hamilton.

Hellmann, Kai-Uwe 2003. Soziologie der Marke. Frankfurt a. M.: Suhrkamp.

Hirschle, Jochen 2012 a. »Religiöser Wandel in der Konsumgesellschaft «, in: Soziale Welt 63, S. $141-162$.

Hirschle, Jochen 2012 b. Die Entstehung des transzendenten Kapitalismus. Konstanz: UVK.

Honneth, Axel 2011. Das Recht der Freiheit. Grundriss einer demokratischen Sittlichkeit. Berlin: Suhrkamp.

Klein, Naomi 2001. No logo. No space, no choice, no jobs. London: Flamingo.

Knoblauch, Hubert 2009. Populäre Religion. Auf dem Weg in eine spirituelle Gesellschaft. Frankfurt a. M.: Campus.

Kocka, Jürgen 1975. Unternehmer in der deutschen Industrialisierung. Göttingen: Vandenhoek \& Ruprecht.

Luckmann, Thomas 1993. Die unsichtbare Religion. 2. Auflage. Frankfurt a. M.: Suhrkamp.

Mayntz, Renate; Scharpf, Fritz W. 1995. »Der Ansatz des akteurszentrierten Institutionalismus ", in Gesellschaftliche Selbstregelung und politische Steuerung, hrsg. v. Mayntz, Renate; Scharpf, Fritz W., S. 39-72. Frankfurt a. M.: Campus.

McCracken, Grant D. 1985. "The trickle down theory rehabilitated «, in The psychology of fashion, hrsg. v. Solomon, Michael R., S. 39-54. New York: Lexington Books.

Leviathan, 40. Jg., 4/2012 
McCracken, Grant D. 1988. Culture and consumption. Bloomington: Indiana University Press.

McKendrick, Neil 1997. "Ursprünge der Konsumgesellschaft ", in Europäische Konsumgeschichte. Zur Gesellschafts- und Kulturgeschichte des Konsums (18.-20. Jahrhundert), hrsg. v. Siegrist, Hannes; Kaelble, Hartmut; Kocka, Jürgen, S. 75-108. Frankfurt a. M.: Campus.

McKendrick, Neil; Brewer, John; Plumb, John H. 1982. The birth of a consumer society: the commercialization of eighteen century England. London: Europa Publications.

Packard, Vance 1957. Die geheimen Verführer. Der Griff nach dem Unbewußten in Jedermann. Frankfurt a. M.: Ullstein.

Schor, Juliet B. 1998. The overspent American. Why we want what we don't need. New York: Harper Perennial.

Schrage, Dominik 2009. Die Verfügbarkeit der Dinge. Eine historische Soziologie des Konsums. Frankfurt a. M.: Campus.

Schulze, Gerhard 1993. Die Erlebnisgesellschaft. Kultursoziologie der Gegenwart. Frankfurt a. M.: Campus.

Seligman, Edwin R. A. 1927. The economics of installment selling. A study in consumer's credit with special reference to the automobile. New York: Harper \& Brothers.

Simmel, Georg 1989 [1900]. Philosophie des Geldes. Gesamtausgabe, Bd. 6. Frankfurt a. M.: Suhrkamp.

Simmel, Georg 1995 [1905]. Philosophie der Mode. Gesamtausgabe, Bd. 10, S. 7-38. Frankfurt a. M.: Suhrkamp.

Stehr, Nico 2007. Die Moralisierung der Märkte. Eine Gesellschaftstheorie. Frankfurt a. M.: Suhrkamp.

Tanner, Jakob 2009. »Konsumtheorien in der Wirtschaftswissenschaft", in Die Konsumgesellschaft in Deutschland 1890-1990. Ein Handbuch, hrsg. v. Haupt, Heinz-Gerhard; Torp, Claudius, S. 335-354. Frankfurt a. M.: Campus.

Turner, Bryan S. 2011. Religion and modern society. Citizenship, secularization and the state. Cambridge: Cambridge University Press.

Ullrich, Wolfgang 2006. Haben wollen. Wie funktioniert die Konsumkultur? Frankfurt a. M.: Fischer.

Zick-Varul, Matthias 2004. Geld und Gesundheit. Konsum als Transformation von Geld in Moral. Berichte aus dem DFG-Graduiertenkolleg »Lebensstile, soziale Differenzen, Gesundheitsförderung «. Eberhard-Karls-Universität Tübingen.

Zick-Varul, Matthias 2009. "Ethical consumption: the case of fair trade", in Wirtschaftssoziologie. Sonderheft 49 der Kölner Zeitschrift für Soziologie und Sozialpsychologie, hrsg. v. Beckert, Jens; Deutschmann, Christoph, S. 366-385. Wiesbaden: VS Verlag für Sozialwissenschaften. 
Zusammenfassung: Die meisten Konsumsoziologen sind sich heute darin einig, dass der moderne Erlebniskonsum und seine Dynamik zur Bildung sozialer Identitäten beitragen und in diesem Sinn eine »moralische " Funktion erfüllen. Eine Gegenposition dazu hat Daniel Bell mit seiner Kritik an den sozial desintegrativen Wirkungen des Konsumhedonismus eingenommen. Der Beitrag zeigt, dass beide Positionen sich miteinander vereinbaren lassen, wenn man die Konsumentenrolle nicht länger isoliert, sondern in ihrem Zusammenhang mit den Arbeitsrollen und den sozialen Mobilitätsprozessen der Konsumenten analysiert. In Anlehnung an das klassische »trickle down «-Konzept wird ein Mehrebenenmodell der Konsumdynamik entwickelt, das zwischen Anerkennung, Disziplinierung und Distinktion als moralischen Funktionen des Konsums unterscheidet.

Stichworte: Erlebniskonsum, Warenästhetik, Religion, soziale Identität, Mehrebenenanalyse, soziale Mobilität, Anerkennung, Disziplinierung, Distinktion

\section{The dynamics of consumption and its impact on the moral integration of modern societies: a conceptual suggestion}

Summary: Most sociologists of consumption agree that modern hedonistic consumerism and its dynamics have an impact on the formation of social identities and are a »moral « phenomena in this sense. Contrary to this, Daniel Bell emphasises the socially desintegrative effects of consumerism. The article shows that both positions are compatible with each other if consumption roles are no longer analyzed in isolation from consumer work roles and processes of social mobility. Following the well known »trickle down « concept, the author suggests a multilevel model of consumption dynamics which distinguishes recognition, social control und distinction as moral functions of consumption.

Keywords: consumerism, hedonism, religion, social identity, multilevel analysis, social mobility, recognition, social control, distinction

Autor

Prof. (em.) Dr. Christoph Deutschmann

Universität Tübingen

Institut für Soziologie

Wilhelmstraße 36

72074 Tübingen

christoph.deutschmann@uni-tuebingen.de

Leviathan, 40. Jg., 4/2012 https://doi.org/10.5771/0340-0425-2012-4-517 